\title{
Self-irradiation-induced disorder in $\left(\mathrm{U}^{238} \mathrm{Pu}\right) \mathrm{O}_{2}$
}

\author{
E. De Bona ${ }^{1,2, *}$, J-Y. Colle $^{1}$, O. Dieste ${ }^{1}$, M. Cologna ${ }^{1}$, T. Wiss $^{1, *}$, G. Baldinozzi ${ }^{2}$, R.J.M. Konings ${ }^{1}$ \\ ${ }^{1}$ European Commission, Joint Research Centre, P.O. Box 2340, 76125 Karlsruhe, Germany \\ ${ }^{2}$ Université Paris-Saclay, Laboratoire Structures, Propriétés et Modélisation des Solides, CNRS, \\ CentraleSupélec, 91190 Gif-sur-Yvette, France \\ *Corresponding authors: \\ Emanuele De Bona, Emanuele.DE-BONA@ext.ec.europa.eu
}

Thierry Wiss, Thierry.WISS@ec.europa.eu

\section{Abstract}

${ }^{238} \mathrm{Pu}$-doped $\mathrm{UO}_{2}$ was characterised periodically to investigate the long-term effects of $\alpha$-self-irradiation on spent nuclear fuel. Samples of two compositions (1.25 and $5 \%$ wt $\left.{ }^{238} \mathrm{Pu}\right)$, were studied by means of X-ray diffraction (XRD), Raman spectroscopy, and transmission electron microscopy (TEM). The cumulated dose received by the samples was measured in displacements per atom (dpa) to allow comparison between the two samples and to the literature values. XRD characterization showed that the lattice swelling approached saturation at $0.3 \%$ of the lattice parameter of the cubic cell at 0.4 dpa damage accumulation. Microstrain decreased for the whole duration of the study, as a result of point defect recombination into dislocation loops, that were observed by TEM for dpa as low as 0.072. Raman highlighted the broadening of the $T_{2 g}$ band, and the increase and broadening of the defect triplet band. Both batches of samples followed the same trend if plotted against dpa, proving that for these dopant concentrations the self-irradiation effects do not depend on the rate of radiation damage creation in the matrix. 


\section{Introduction}

During irradiation of nuclear fuel (NF) in a nuclear reactor, fission products (FPs), minor actinides (MAs), and plutonium are generated and remain embedded in the fuel matrix. While FPs are mostly short-lived $\beta$ emitters, MAs and Pu are long-lived $\alpha$-emitters and will keep depositing their energy in the spent NF (SNF) for thousands of years. As a result, radiation damage and radiogenic He will accumulate in the SNF matrix, influencing its properties over very long time periods.

Currently, two main solutions are implemented for the handling of SNF: reprocessing for the production of mixed-oxide (MOX) fuel and disposal of high level waste (HLW), or direct disposal in a deep geological repository ${ }^{1}$. In both cases, a period of interim storage follows the discharge from the reactor. Changes in the SNF properties induced by self-irradiation, such as lattice swelling, loss of integrity, thermal conductivity decrease, or He build-up (over-pressurization of the fuel rod) must be evaluated in to support safety assessment of, both interim storage and the final repository ${ }^{2,3}$.

However, due to the extremely different temperature and radiation conditions between reactor irradiation and storage, the analysis of SNF after discharge from the reactor is not accurate enough to predict the status of SNF after thousands of years of self-irradiation. Moreover, due to its chemical and microstructural inhomogeneities, SNF is a very complex system to study, besides being a hazardous material to handle.

A way to circumvent these issues is the setup of separate effect studies, that allow studying simpler surrogate systems from the compositional point of view, with the use of SIMfuel ${ }^{4-6}$, or for the radiation damage aspect, by using ion implantation ${ }^{7-9}$ or $\alpha$-doped materials ${ }^{10-12}$. These latter are especially preferable when investigating $\alpha$-self-irradiation effects, where the overlapping of ballistic (recoil nucleus) and electronic ( $\alpha$ particle) energy losses partially heals the overall radiation damage inflicted to the matrix ${ }^{13,14}$.

In this work, the $\alpha$-self-irradiation effect on the $\mathrm{SNF} \mathrm{UO}_{2}$ matrix has been studied by periodically characterising $\left(\mathrm{U}_{\mathrm{x}}{ }^{238} \mathrm{Pu}_{1-\mathrm{x}}\right) \mathrm{O}_{2}$ samples by means of X-Ray Diffraction (XRD), Raman spectroscopy, and Transmission Electron Microscopy (TEM). The combination of these systematic characterizations allowed investigating how the overlapping of electronic and nuclear energy losses during $\alpha$-self-irradiation leads to 
structural disorder, lattice swelling, and strain relaxation as a consequence of point defects clustering into extended defects as dislocation loops. 


\section{Experimental work}

The whole experimental work, as well as the data analysis, were performed at the European Commission Joint Research Centre (JRC) in Karlsruhe (Germany). Due to the high radiotoxicity of the samples, both their preparation and characterisation were entirely performed inside shielded gloveboxes for the handling of radioactive materials.

\subsection{Sample preparation}

The sample preparation was already described extensively in a separate publication ${ }^{15}$. Briefly, a Pu reactant containing $53 \%$ wt ${ }^{238} \mathrm{Pu}$ was dissolved in $\mathrm{HNO}_{3}$ and mixed in the desired ratios with a uranyl nitrate solution of known concentration. The two compositions targeted were 2.5 and $10 \% \mathrm{Pu}$-doped $\mathrm{UO}_{2}$, meaning an actual

${ }^{238} \mathrm{Pu}$ content of about 1.25 and $5 \%$. The higher dopant concentration was chosen in order to be able to observe a complete saturation of the lattice swelling during the timeframe of this study ( 2 years). On the other hand, the lower dopant concentration was chosen to have slower evolution of the kinetics, and therefore better observation of the early stages of self-irradiation. For comparison, in terms of activity real SNF is close to a $0.1 \%$ wt ${ }^{238} \mathrm{Pu}$-doped $\mathrm{UO}_{2}$. Precipitation was triggered by ammonia addition, and the resulting precipitates were washed in distilled water, then calcined first under simulated air $\left(80 \% \mathrm{~N}_{2}-20 \% \mathrm{O}_{2}\right)$ at $400{ }^{\circ} \mathrm{C}$, and finally under $\mathrm{Ar}-4 \% \mathrm{H}_{2}$ gas mixture at $700{ }^{\circ} \mathrm{C}$. The $(\mathrm{U}, \mathrm{Pu}) \mathrm{O}_{2}$ powders were then pressed in $5 \mathrm{~mm}$ diameter dies under $738 \mathrm{MPa}$ and sintered at the nominal temperature of $1650{ }^{\circ} \mathrm{C}$ under $\mathrm{Ar}-4 \% \mathrm{H}_{2}$ for $6 \mathrm{~h}$. The final disks had average geometrical and Archimedes relative densities of 94.7 and $97.5 \%$ of the theoretical density (TD) respectively. The chemical compositions of these samples were assessed by the lattice parameters measured using XRD and were found to be in excellent agreement with the targeted ones.

\subsection{Dose calculation}

The dose received by the samples was quantified by using the displacement per atom (dpa). This unit accounts for the atoms displaced from their original lattice sites as a consequence of the ballistic collisions 
generated by the $\alpha$-decays taking place in the material. It allows comparing radiation damage generated by different sources, although it does not take into consideration defect recombination. The number of displaced atoms per $\alpha$-decay was calculated using SRIM ${ }^{16}$. A value of 1650 displacements was obtained considering 20 and $40 \mathrm{eV}$ displacement energies thresholds for the oxygen and the uranium atoms respectively ${ }^{17}$. A simplified formula for the dpa calculation, highlighting the linear dependence on the dopant concentration $\left(m_{\text {dopant }} / m_{\text {sample }}\right)$, is the following:

$$
d p a=\frac{1650 \cdot a \cdot t \cdot M m}{3 \cdot N_{A}} \cdot \frac{m_{\text {dopant }}}{m_{\text {sample }}}
$$

In this equation, $a$ is the specific activity of the $\alpha$-emitter $\left[\mathrm{g}^{-1} \mathrm{~s}^{-1}\right], t$ is the irradiation time $[\mathrm{s}], N_{A}$ is the Avogadro number $\left[\mathrm{mol}^{-1}\right]$, and $\mathrm{Mm}$ is the molar mass $\left[\mathrm{g} \mathrm{mol}^{-1}\right]$ of the sample $\left(\mathrm{UO}_{2}\right.$ molar mass can be used, considering the low dopant concentration, and the fact the dopant consists largely of isobaric $\left.{ }^{238} \mathrm{Pu}\right)$. The derivation of this formula was described in another publication on related topics ${ }^{15}$.

\subsection{Characterization}

The as-sintered disks were characterised by means of SEM and XRD in order to assess their microstructural characteristics and structural homogeneity. The samples selected for the periodic characterisation were annealed at the sintering temperature for $2 \mathrm{~h}$ to heal the radiation damage accumulated in the time between the synthesis and the beginning of the characterisation campaign. Raman characterization was performed periodically on the same dedicated samples, while a new fragment was taken for XRD and TEM analyses (from a sample of the same batch) and prepared for each measurement at each different dose level. Samples of both compositions were always analysed on the same day. During the whole ageing study, samples were stored under the gloveboxes $\mathrm{N}_{2}$ atmosphere at room $\mathrm{T}$.

Samples for XRD characterisation were dispersed in isopropanol, ground manually, and poured onto flat Si(9 1 1) sample holders. Measurements were performed using a Bruker D8 diffractometer mounted in $\Theta-2 \Theta$ Bragg-Brentano geometry, a ceramic copper tube (40 kV, $40 \mathrm{~mA}$ ) generator with a curved Ge $(1,1,1)$ primary monochromator, and equipped with a LinxEye detector. Data were analysed with the software Jana2006 ${ }^{18}$, 
using Pseudo-Voigt functions for peak fitting. The lattice parameter was determined by Rietveld refinement, while microstrain and crystallite size were calculated using the Williamson-Hall approach ${ }^{19}$. A LaB 6 reference sample was used for the calibration of the device.

Raman characterisation was performed using a Horiba Jobin-Yvon T 64000 spectrometer, equipped with a 1800 grooves/mm grating, a low noise $\mathrm{LN}_{2}$ cooled CCD detector, and an edge filter to block the laser elastic scattering coming from the sample. A long working distance $(10.6 \mathrm{~mm})$ objective, offering a 0.5 numerical aperture with a X50 magnification, was mounted on the device. Acquisitions were performed in the $200-$ $1300 \mathrm{~cm}^{-1}$ range, using a $647 \mathrm{~nm} \mathrm{Kr}{ }^{+}$excitation laser. Before each measurement, the spectrograph was calibrated with the $\mathrm{T}_{2 \mathrm{~g}}$ excitation of a Si single crystal $\left(520.5 \mathrm{~cm}^{-1}\right)$. Since the instrument is not included in a glovebox, the radioactive sample that was encapsulated in a special sample holder designed at the JRC Karlsruhe ${ }^{20}$.

Samples for TEM characterisation were prepared by crushing method ${ }^{21}$ : fragments of a few milligrams were milled in a mortar, suspended in methanol, and dripped over a 400 mesh copper grid coated with carbon. Analyses were performed in a $\mathrm{FEI} \mathrm{I}^{\mathrm{TM}}$ Tecnai $\mathrm{G} 2$ equipped with a field emission gun. This instrument was adapted for the study of radioactive materials and a small glovebox was attached to it for the loading and unloading of the samples ${ }^{22}$. 


\section{Results and discussion}

\subsection{X-Ray Diffraction}
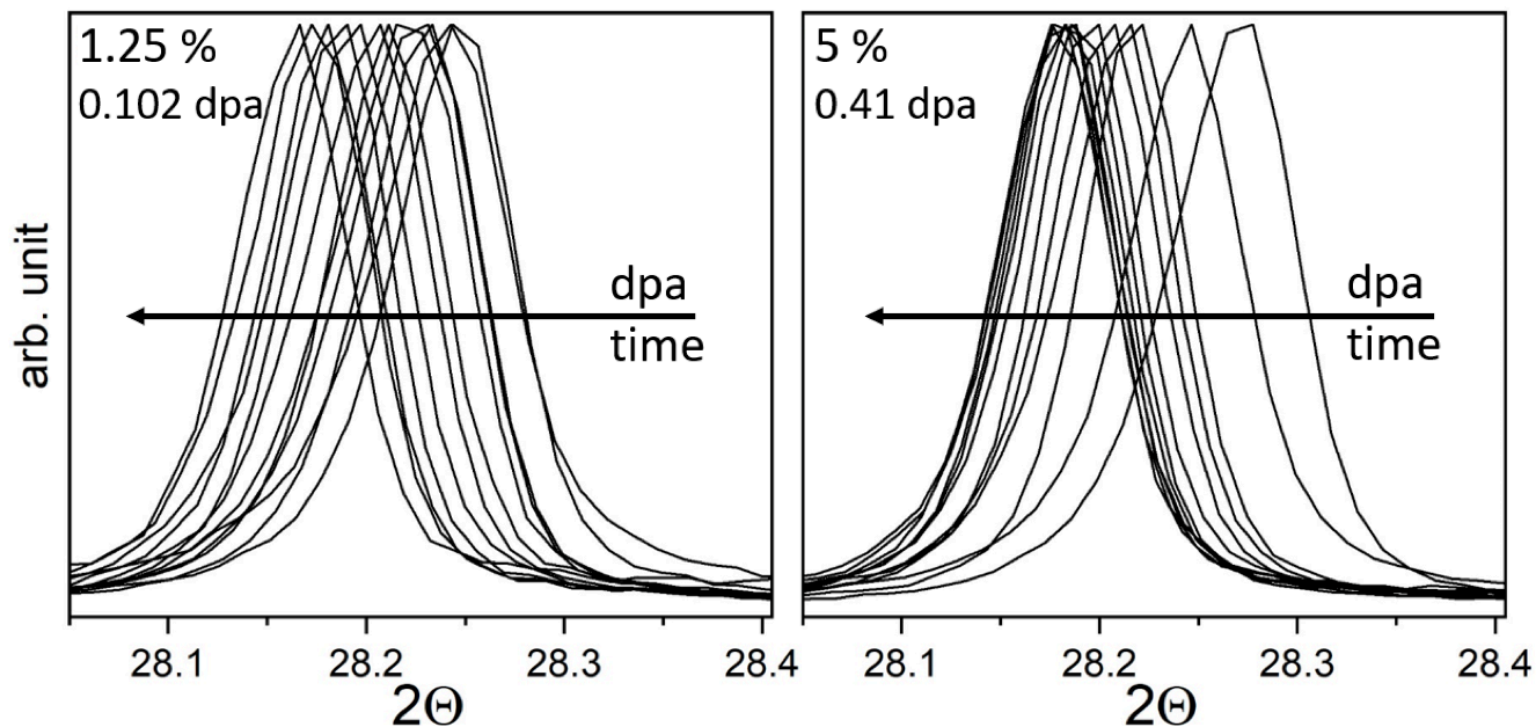

Figure 1: Self-irradiation-induced XRD (111) peak shift. Both samples were analyzed on the same days. Measurements ranged from day 0 to day 564, meaning 0.102 and 0.41 dpa for the 1.25 and $5 \%$ samples respectively.

Radiation-induced lattice swelling results in a shift of the XRD peaks towards lower angles. Figure 1 shows the shift of the (111) reflection peak for samples of both compositions, ranging from day 0 (annealing day) to day 564 ( 0.102 and 0.41 dpa cumulated by the 1.25 and $5 \%$ samples respectively).

It is worth noting that the initial peak position and the amplitude of the shifts (for a given measurement day) both depend on the chemical composition of the samples. In the $5 \%$ Pu substituted sample indeed, the peaks measured at the later times were almost overlapping, suggesting the swelling was approaching its saturation value. This was reflected directly in the lattice parameter calculated by Rietveld refinement. 

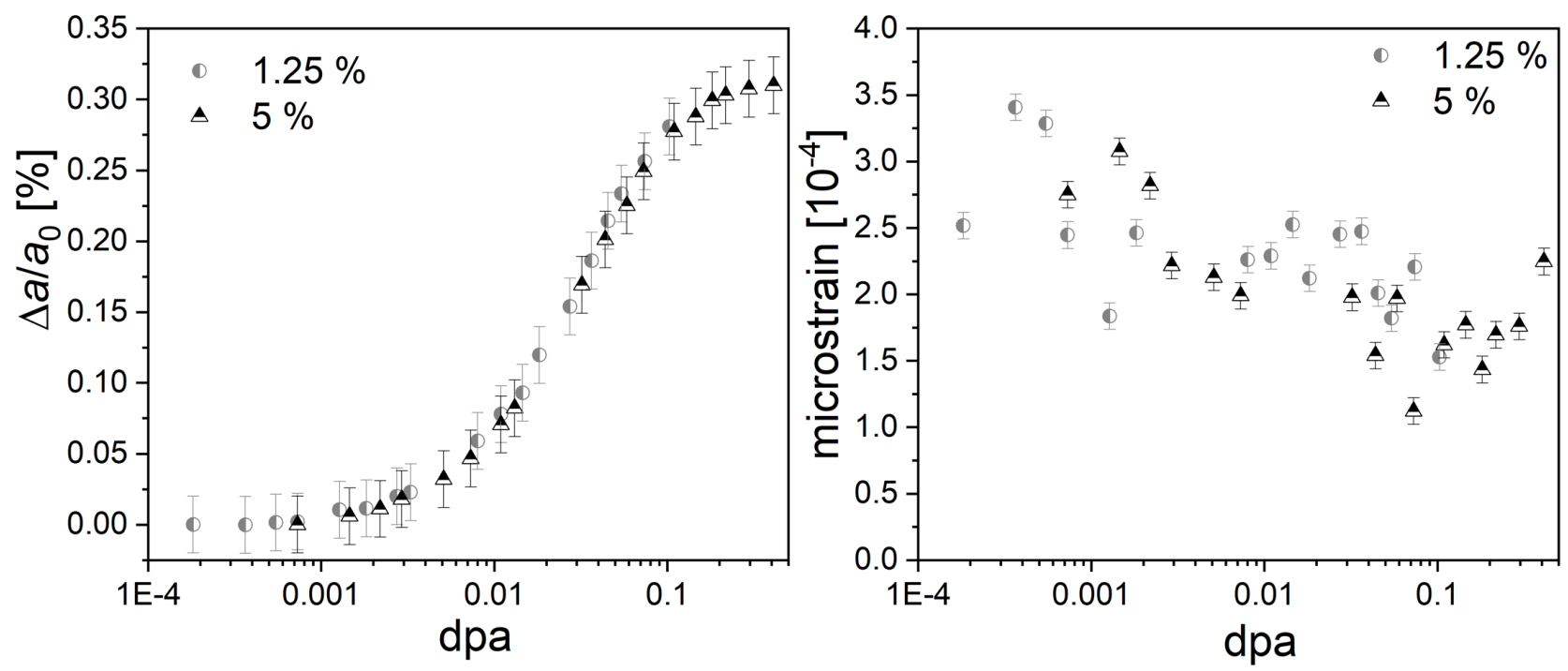

Figure 2: Relative lattice parameter increase $\left(\Delta a / a_{0}\right)$ and microstrain as calculated from the XRD patterns shown in Figure 1.

The relative lattice parameter increase and the microstrain derived from these XRD patterns are shown in Figure 2. In very good agreement with the literature for $\alpha$-self-irradiating $\mathrm{PuO}_{2}{ }^{23},(\mathrm{U}, \mathrm{Pu}) \mathrm{O}_{2}{ }^{24}$, and $(\mathrm{U}, \mathrm{Am}) \mathrm{O}_{2}{ }^{25}$, the relative lattice parameter increase approached saturation at $0.3 \%$ for $0.4 \mathrm{dpa}$. Microstrain, that was already initially very low, decreased even further. Higher $\Delta \mathrm{a}_{\mathrm{a}} \mathrm{a}_{0}$ values were reported for much higher doses (above $1 \mathrm{dpa}$ ) in other works ${ }^{11}$, but the absence of data in the intermediate range does not allow to draw any conclusion on the existence of a second swelling stage yet. Indeed, no measurements were performed between 4 and $100 \mathrm{dpa}$. Considering only the available data up to $4 \mathrm{dpa}$, a saturation value of the lattice parameter of $0.4 \%$ was determined ${ }^{26}$, whereas it reached $0.6 \%$ for $100 \mathrm{dpa}$.

Recent dual beam studies showed a reduction of the strain, accompanied by a microstrain increase, as a result of the superposition of nuclear and electronic energy losses in $\mathrm{UO}_{2}{ }^{13,14}$. Combined low- and high-energy irradiations resulted in the redistribution of the implanted layer of the low-energy irradiation. This was due to the increased defect mobility caused by the thermal spike. However, while point defect recombination can be qualitatively similar in ion beam experiments and in real systems (vacancies transform into voids, interstitials into dislocation loops, as we discuss in Section 3.3), these results are only partially representative of the real case, due to the extrinsic anisotropy and the ion implantation effect.

Our X-ray experiments demonstrate the self-irradiation strain and microstrain fields are perfectly isotropic. Therefore, the observed effect on strain is opposite: as the dose increases, strain in the self-irradiation 
systems always increases while microstrain decreases. Radiation-induced lattice swelling was detectable from the isotropic shift of every XRD reflection (caused by the elastic expansion in each crystallographic direction). As the dose increased, point defects coalesced into extended ones, uniformly populating the matrix with dislocation loops and microcavities. This microstrain (i.e., the strain variance) decrease simultaneous to the strain increase $\left(\Delta a / a_{0}\right)$ can be explained by the linear superposition of isotropically oriented Eshelby's elastic fields ${ }^{27}$. Therefore, the system behaves as an effective medium where the elastic strain field tends to homogenize. Rietveld refinement on samples with different initial activity demonstrate that during self-irradiation, up to $0.4 \mathrm{dpa}$, the relation between strain and dose is linear. This provides evidence that no macroscopic synergistic effects occur from the damage overlap and accumulation, and that a linear superposition effect is confirmed both for the strain and the microstrain.

\subsection{Raman}
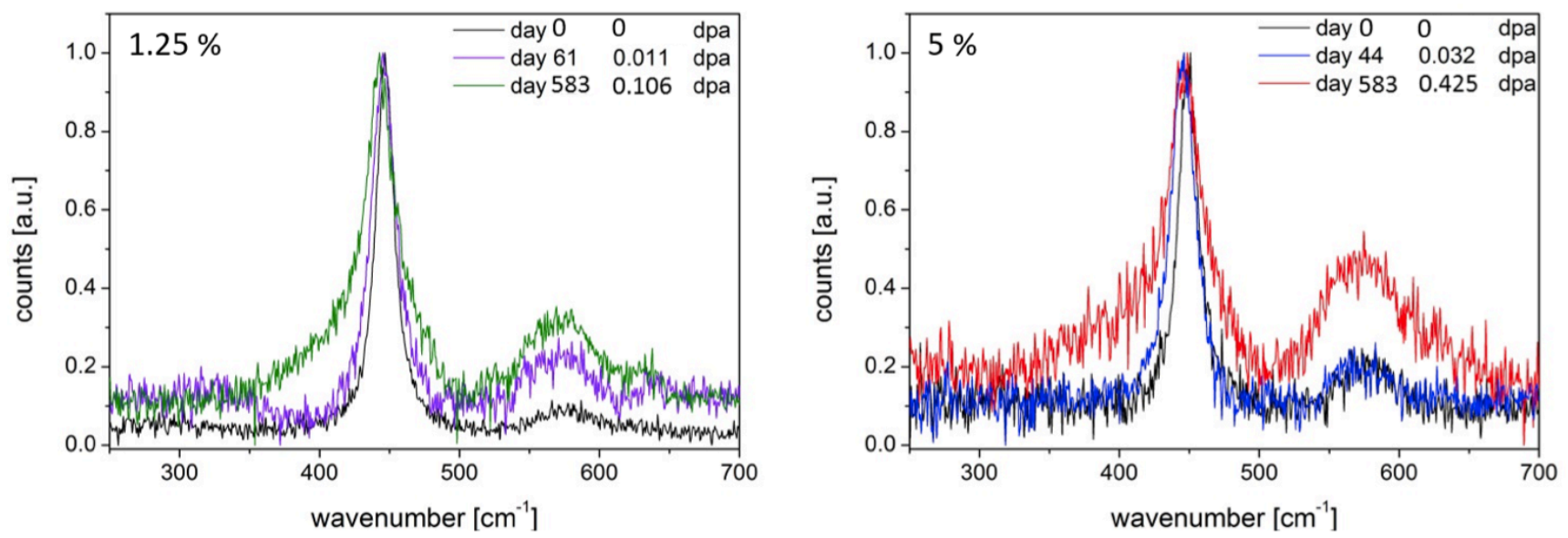

Figure 3: Evolution of the Raman spectra of the two samples from day 0 to day 583 ( 0.106 and 0.425 dpa for 1.25 and $5 \%$ respectively).

The progressive loss of order in the lattice induced by $\alpha$-self-irradiation could be detected also by Raman scattering. The evolution of the spectra from day 0 to day 583 (0.106 and $0.425 \mathrm{dpa}$ for 1.25 and $5 \%$ respectively) is shown in Figure 3. At the start of the data collection, two features were already visible:

- The $T_{2 g}$ band, related to the antiphase oscillation of the $O$ atoms around the metal atom (that is at rest), is the only Raman-active mode of fluorite structures with $\mathrm{MO}_{2}$ chemical formula. Its position depends on the chemical composition of the samples (as the dopant is isobaric): the strength of the $\mathrm{M}-\mathrm{O}$ bond increases with the Pu content, shifting the band to higher wavenumbers ${ }^{28}$. The values of 
$446.8 \mathrm{~cm}^{-1}(1.25 \%)$ and $449.3 \mathrm{~cm}^{-1}(5 \%)$ are in excellent agreement with what reported in the literature for $(\mathrm{U}, \mathrm{Pu}) \mathrm{O}_{2}$ of various compositions ${ }^{29-31}$.

- The LO component of the ungerade $T_{1 u}$ optic mode around $575 \mathrm{~cm}^{-1}$ that, in a perfect lattice, would be only infrared-active. In this case, it became Raman-active due to the loss of selection rules caused by the translation-symmetry breaking introduced by the Pu substitutional atoms in the $\mathrm{U}$ lattice ${ }^{32}$. Its intensity should indeed be roughly proportional to the Pu content in a diluted model, but a quantitative demonstration was not possible due to the higher noise of the spectra of the $5 \%$ substituted samples.

Self-irradiation has an effect on both of these features:

- The $T_{2 g}$ band underwent an asymmetric broadening, caused by the density of vibrational states of this branch outside the centre of the Brillouin zone. This broad component grows in intensity as defects accumulate in the structure producing a progressive loss of crystalline order, therefore resulting in a larger broadening asymmetry of the signal towards low wavenumbers.

- The band around $575 \mathrm{~cm}^{-1}$ increased and broadened. In this case, the effect is due to the combination of the increase of the dopant-induced band $\left(\mathrm{v}_{2}\right)$, and the growth of two additional components possibly related to the radiation defect accumulation $\left(\mathrm{v}_{1}\right.$, at $530 \mathrm{~cm}^{-1}$, and $\mathrm{v}_{3}$, at $\left.630 \mathrm{~cm}^{-1}\right)$ around $\mathrm{it}^{33}$. These three bands constitute the so-called defect triplet band, where the contribution of $v_{1}$ and $v_{3}$ is particularly sensitive to the defect concentrations in the oxygen sublattice (Magneli-type defects) ${ }^{34,35}$.

Unfortunately, the fluorescence signal increased over time, lowering the signal-to-noise ratio of the measurements. The quality of the data decreased also due to the damage induced by $\alpha$-irradiation on the carbon glue of the sample holders. For these reasons, some effects, such as the defect triplet band intensity increase, could be appreciated only qualitatively.

The ratio between the intensities of the softer broad component and the initial $T_{2 g}$ mode is shown in Figure 4 (as apparent $T_{2 g}$ shift). Also in this case the data for the two Pu compositions followed the same trend, hinting towards the linear scaling of this effect with the dose, although with higher scatter, and saturating at 
lower dpa values. As already shown in other recent studies ${ }^{31,33}$, Raman is therefore a suitable technique for studying the $\alpha$-self-irradiation effect in actinide dioxides with fluorite structures.

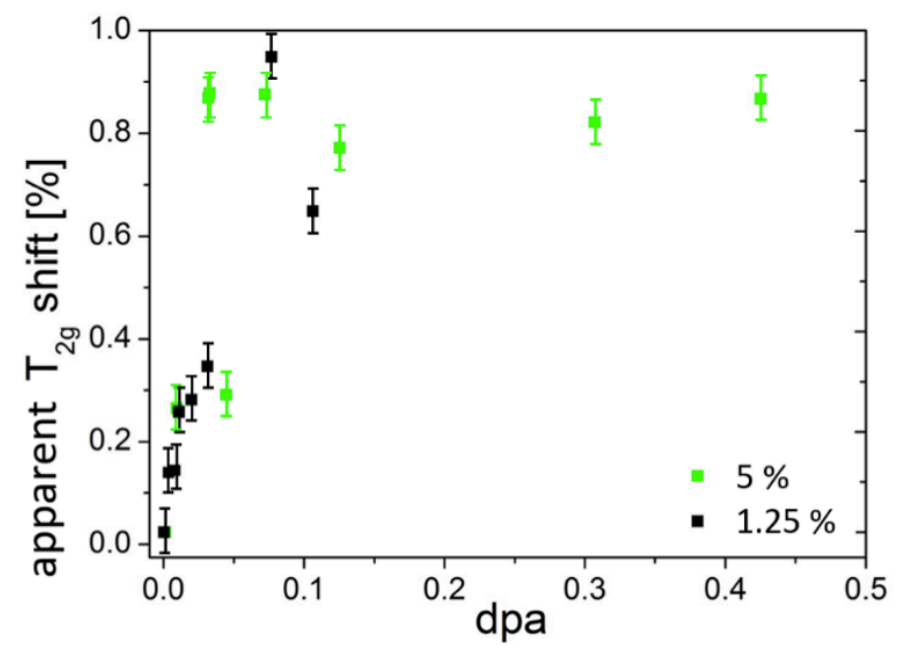

Figure 4: Apparent $T_{2 g}$ shift resulting from the intensity ratio of the low wavenumber component to the initial $T_{2 g}$ intensity.

\subsection{Transmission Electron Microscopy}

Figure 5 shows the results of TEM characterization performed on samples of both compositions for different dpa levels. Extremely small (less than $10 \mathrm{~nm}$ ) dislocation loops were already found in a $1.25 \%$ sample having cumulated $0.031 \mathrm{dpa}$ (170 days). This sample was also used in another study on the self-irradiation-induced thermal conductivity degradation, where the presence of small black dots (low-order point defect ${ }^{36}$ or vacancy ${ }^{37}$ clusters) was also observed by $\mathrm{TEM}^{15}$. At this point, microstrain was already significantly decreased, as shown in Figure 2. With increasing the cumulated dose ( 0.062 and $0.338 \mathrm{dpa}$ in $5 \%$ samples aged 85 and
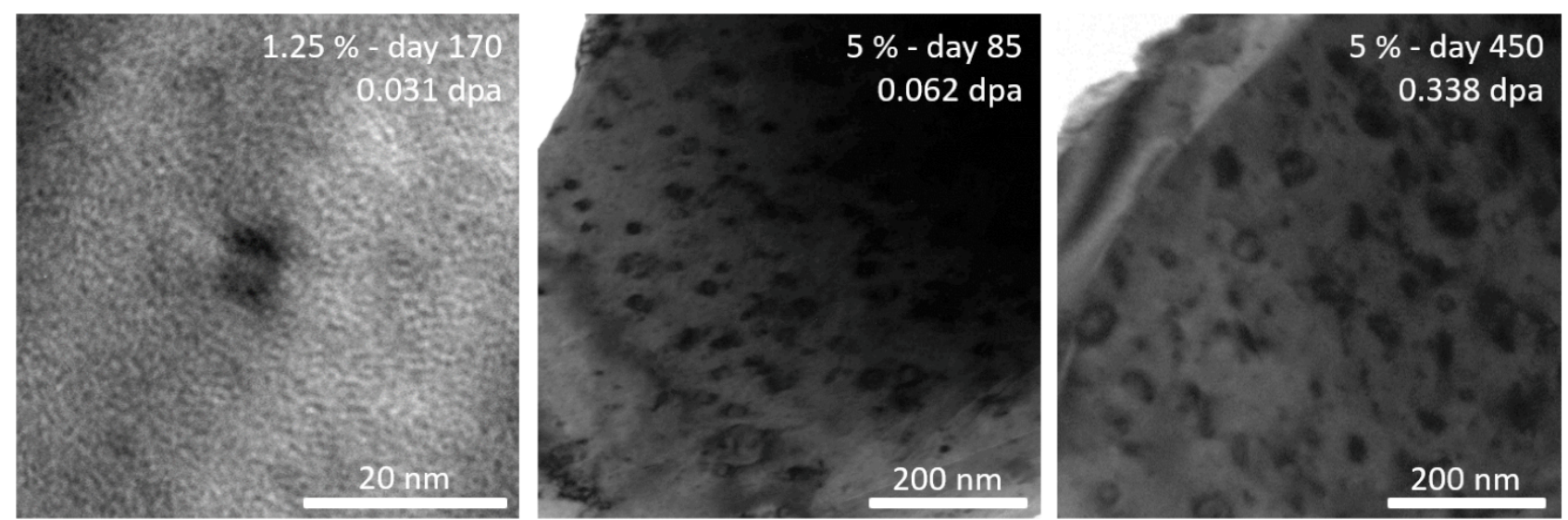

Figure 5: TEM images of samples of the two different compositions at various dpa levels $(0.031 \mathrm{dpa}$ from a $1.25 \%$ sample aged 170 days, and 0.06 and 0.338 dpa from a $5 \%$ sample aged 85 and 450 days). 
450 days), dislocation loops grew in size and density, whereas the microstrain decreased further, although a lower rate as already pointed out in section 3.1.

\subsection{Linearity of the self-irradiation effect and possible extrapolations}

The diffusion-controlled evolution of a microstructure in a closed system is driven by thermally activated motions of point defects. This is dependent on local parameters (i.e., jump frequencies, local structure and defect organization) and non-local effects (described by fields, like the composition, the mechanical stress, and the electric fields).

When systems are maintained far from equilibrium by a perturbation (as irradiation) defects are continually being added to (and removed from) the system. Such systems can be described as open systems. Open systems are never at equilibrium, but assuming that the changes to the system occur on a time-scale that is faster than the rate at which the system returns to equilibrium following the perturbation, the system could eventually evolve asymptotically into a stable configuration. Such stable, non-equilibrium systems are referred as steady-state systems: they can only be maintained at the cost of non-vanishing currents (fluxes) of thermodynamic resources. Following Prigogine's assumption, these systems are characterized by a nonvanishing entropy production rate that is function of the fluxes of the various irreversible processes occurring in the system and the associated generalized forces (gradients of temperature, chemical potentials, ...) as in Onsager's reciprocity relations.

The fluxes induced by radioactive decay or by external irradiation, become the relevant variable to describe the irradiated system, and they can lead to qualitatively different steady states of the system. In general, the behaviour of these systems out of equilibrium is not linear, and therefore, the linear extrapolation of the properties of these systems cannot be assumed. This restriction is for instance illustrated by the existence of a homeostatic range in biological systems. Therefore, the description of the steady states of a crystal undergoing irradiation cannot be properly parametrized by the radiation dose (fluence) making abstraction of the radiation flux, unless there is actual proof that the out of equilibrium system has a linear behaviour for the range of fluxes employed in the experiment. One example of that is provided by the appearance of 
distinct compositional patterns in systems driven by competing dynamics at different length scales that are responsible for different mechanical behaviours of the system ${ }^{38}$.

Now the current paper provides the demonstration that the system behaves linearly for different values of the flux. But it is not possible to extend this claim to irradiation experiments performed by other groups or to generalize this assumption to previous results. This can explain why different results for the saturation effects can be observed in experiments carried on with higher fluxes, where a different steady state can be reached, leading eventually to different properties.

The question underpinning this discussion is then which system is a better benchmark for predicting the longterm behaviour of spent nuclear fuels, where the activity of the systems (flux) fells in a particular range Nevertheless, it can be pointed out that the present work is in good agreement with previous studies, that showed a similar linear behavior for decay rates comparable to those studied here (namely 0.1 and $10 \% \mathrm{wt}$ ${ }^{238} \mathrm{Pu}$-doped $\left.\mathrm{UO}_{2}\right)^{11}$. A $0.1 \% \mathrm{wt}^{238} \mathrm{Pu}$-doped $\mathrm{UO}_{2}$ is representative of irradiated fuel 10 years after discharge, for which the activity remains equivalent for centuries. Considered the linearity of the measured properties, it can be stipulated that compounds with a higher decaying rate (up to 100 times higher) offer a good representation of the effect of $\alpha$-self-irradiation in spent fuel at equivalent dose. 


\section{Conclusion}

In this work, $\mathrm{UO}_{2}$ doped with ${ }^{238} \mathrm{Pu}$ was used to simulate spent nuclear fuel ageing. The samples were characterised periodically by XRD, Raman, and TEM, providing a complete and systematic set of data. Two different compositions were chosen, in order to be able to observe the saturation of the self-irradiationinduced lattice swelling during the timeframe of the study (2 years), but at the same time to have slow and therefore easily observable evolution of the damage kinetics. This allowed to prove that, the radiationinduced disorder was not affected by the dose-rate for these low dopant concentrations ( 1.25 and $5 \% \mathrm{wt}$ ).

XRD characterisation confirmed the relative lattice parameter increase of $0.3 \%$ was reached at $0.4 \mathrm{dpa}$, as already reported in the literature. Microstrain was also calculated from the XRD measurements by applying the Williamson-Hall approach, and it was found to decrease slowly throughout the whole duration of the study. Raman characterization highlighted three main $\alpha$-self-irradiation effects: the asymmetric broadening of the $T_{2 g}$ mode, the increase and broadening of the $T_{1 u}$ band, and of the defect triplet band. The latter observation was only qualitative, due to the increasing noise in the measurements coming from the irradiated sample environment.

The measured phenomena (lattice swelling, microstrain relaxation, $T_{2 g}$ broadening) were all found to be increasing functions of the cumulated dose, independently from the initial activity of the samples (for the two dopant concentrations used in this work). No macroscopic synergistic effect on the strain and microstrain related to damage overlap and dose accumulation was observed. This means that using strong $\alpha$-emitters (such as ${ }^{238} \mathrm{Pu}$ ) can be a good solution to assess long-term $\alpha$-self-irradiation effects within a timeframe suited for laboratory studies.

It is worth pointing out that real SNF, due to the fission product accumulation and the extreme radiation environment experienced in the reactor, differs significantly from undamaged $\left(\mathrm{U}_{x}{ }^{238} \mathrm{Pu}_{1-x}\right) \mathrm{O}_{2}$. However, the centre of the fuel reaches high temperatures during operation, and therefore this region of pellets is relatively damage- and impurity-free also in the spent nuclear fuel. In this part of SNF, the contribution of $\alpha$ - 
self-irradiation can then be significantly similar to the one studied in these experiments and it can affect the overall properties of the material in the way investigated by this study.

Moreover, also the storage temperature of the samples used in this study differs from that of real SNF in wet storage conditions (about $200{ }^{\circ} \mathrm{C}$ ). Further studies are needed to assess the differences in the defect accumulation and recombination kinetics between room $\mathrm{T}$ and wet storage conditions $\mathrm{T}$.

\section{Acknowledgements}

The work was performed in the framework of the EURATOM Research and Training Program 2014-2018 and extension 2019-2020, through a European Commission PhD grant.

The authors would like to acknowledge D. Bouexiere, J. Boshoven, S. Stohr, and H. Hein for the valuable support both in the preparation and characterization of the samples.

\section{Conflict of interest statement}

On behalf of all authors, the corresponding author states that there is no conflict of interest.

\section{Data availability statement}

The datasets generated during and/or analyzed during the current study are available from the corresponding author on reasonable request. 


\section{References}

1. Status and Trends in Spent Fuel and Radioactive Waste Management. (INTERNATIONAL ATOMIC ENERGY AGENCY, 2018).

2. Ahn, T., Rondinella, V. \& Wiss, T. Potential stress on cladding imposed by the matrix swelling from alpha decay in high burnup spent nuclear fuel. 14th Int. High-Level Radioact. Waste Manag. Conf. IHLRWMC 2013 Integr. Storage, Transp. Dispos. 1, 111-117 (2013).

3. Staicu, D. et al. Impact of auto-irradiation on the thermophysical properties of oxide nuclear reactor fuels. J. Nucl. Mater. 397, 8-18 (2010).

4. Lucuta, P. G., Verrall, R. A., Hastings, I. J. \& Matzke, H. Thermal conductivity and gas release from SIMFUEL. (1993).

5. Lucuta, P. G., Verrall, R. A., Matzke, H. \& Palmer, B. J. Microstructural features of SIMFUEL - Simulated high-burnup $\mathrm{UO}_{2}$-based nuclear fuel. J. Nucl. Mater. 178, 48-60 (1991).

6. Wangle, T., Tyrpekl, V., Cologna, M. \& Somers, J. Simulated $\mathrm{UO}_{2}$ fuel containing Csl by spark plasma sintering. J. Nucl. Mater. 466, 150-153 (2015).

7. Garrido, F., Vincent, L., Nowicki, L., Sattonnay, G. \& Thomé, L. Radiation stability of fluorite-type nuclear oxides. Nucl. Instruments Methods Phys. Res. Sect. B Beam Interact. with Mater. Atoms 266, 2842-2847 (2008).

8. Marchand, B. et al. Xenon migration in $\mathrm{UO}_{2}$ under irradiation studied by SIMS profilometry. J. Nucl. Mater. 440, 562-567 (2013).

9. Gutierrez, G., Onofri, C., Miro, S., Bricout, M. \& Leprêtre, F. Effect of ballistic damage in $\mathrm{UO}_{2}$ samples under ion beam irradiations studied by in situ Raman spectroscopy. Nucl. Instruments Methods Phys. Res. Sect. B Beam Interact. with Mater. Atoms 434, 45-50 (2018).

10. Prieur, D. et al. Self-irradiation effects in dense and tailored porosity $U_{1-y} A m_{y} O_{2-x}(y=0.10 ; 0.15)$ 
compounds. J. Nucl. Mater. 411, 15-19 (2011).

11. Wiss, T. et al. Evolution of spent nuclear fuel in dry storage conditions for millennia and beyond. J. Nucl. Mater. 451, 198-206 (2014).

12. Talip, Z. et al. Thermal diffusion of helium in ${ }^{238} \mathrm{Pu}$-doped $\mathrm{UO}_{2}$. J. Nucl. Mater. 445, 117-127 (2014).

13. Gutierrez, G., Gosset, D., Bricout, M., Onofri, C. \& Debelle, A. Effect of coupled electronic and nuclear energy deposition on strain and stress levels in $\mathrm{UO}_{2}$. J. Nucl. Mater. 519, 52-56 (2019).

14. Bricout, M. et al. Radiation damage in uranium dioxide: Coupled effect between electronic and nuclear energy losses. J. Nucl. Mater. 531, 151967 (2020).

15. De Bona, E. et al. Radiation effects in alpha-doped $\mathrm{UO}_{2}$. Nucl. Instruments Methods Phys. Res. Sect. $B$ Beam Interact. with Mater. Atoms 468, (2020).

16. Ziegler, J. F., Ziegler, M. D. \& Biersack, J. P. SRIM - The stopping and range of ions in matter (2010). Nucl. Instruments Methods Phys. Res. Sect. B Beam Interact. with Mater. Atoms 268, 1818-1823 (2010).

17. Soullard, J. \& Alamo, E. A. Etude du ralentissement des ions dans une cible diatomique. Radiat. Eff. 38, 133-139 (1978).

18. Petříček, V., Dušek, M. \& Palatinus, L. Crystallographic Computing System JANA2006: General features. Zeitschrift für Krist. - Cryst. Mater. 229, 345 (2014).

19. Williamson, G. . \& Hall, W. . X-ray line broadening from filed aluminium and wolfram. Acta Metall. 1, 22-31 (1953).

20. Naji, M. et al. An original approach for Raman spectroscopy analysis of radioactive materials and its application to americium-containing samples. J. Raman Spectrosc. 46, 750-756 (2015).

21. Wiss, T. et al. TEM study of alpha-damaged plutonium and americium dioxides. J. Mater. Res. 30, 1544-1554 (2015). 
22. Wiss, T. et al. Recent Results of Microstructural Characterization of Irradiated Light Water Reactor Fuels using Scanning and Transmission Electron Microscopy. JOM 64, 1390-1395 (2012).

23. Chikalla, T. D. \& Turcotte, R. P. Self-radiation damage ingrowth in ${ }^{238} \mathrm{PuO}_{2}$. Radiat. Eff. 19, 93-98 (1973).

24. Kato, M. et al. Self-radiation damage in plutonium and uranium mixed dioxide. J. Nucl. Mater. 393, 134-140 (2009).

25. Horlait, D., Lebreton, F., Roussel, P. \& Delahaye, T. XRD Monitoring of $\alpha$ Self-Irradiation in UraniumAmericium Mixed Oxides. Inorg. Chem. 52, 14196-14204 (2013).

26. Weber, W. J. et al. Plutonium in Waste Forms. Plutonium Handb. 2349-2422 (2019).

27. Eshelby, J. D. The determination of the elastic field of an ellipsoidal inclusion, and related problems. Proc. R. Soc. London. Ser. A. Math. Phys. Sci. 241, 376-396 (1957).

28. Jégou, C. et al. Raman spectroscopy characterization of actinide oxides $\left(\mathrm{U}_{1-\mathrm{y}} \mathrm{Pu}_{\mathrm{y}}\right) \mathrm{O}_{2}$ : Resistance to oxidation by the laser beam and examination of defects. J. Nucl. Mater. 405, 235-243 (2010).

29. Talip, Z. et al. Raman microspectroscopic studies of unirradiated homogeneous $\left(\mathrm{U}_{0.76} \mathrm{Pu}_{0.24}\right) \mathrm{O}_{2+\times}$ : the effects of Pu content, non-stoichiometry, self-radiation damage and secondary phases. J. Raman Spectrosc. 48, 765-772 (2017).

30. Elorrieta, J. M. et al. Raman study of the oxidation in $(\mathrm{U}, \mathrm{Pu}) \mathrm{O}_{2}$ as a function of Pu content. J. Nucl. Mater. 495, 484-491 (2017).

31. Medyk, L. et al. Determination of the plutonium content and $\mathrm{O} / \mathrm{M}$ ratio of $(\mathrm{U}, \mathrm{Pu}) \mathrm{O}_{2-x}$ using Raman spectroscopy. J. Nucl. Mater. 541, 152439 (2020).

32. Dolling, G., Cowley, R. A. \& Woods, A. D. B. The crystal dynamics of uranium dioxide. Can. J. Phys. 43, 1397-1413 (1965).

33. Villa-Aleman, E., Houk, A. L., Bridges, N. J. \& Shehee, T. C. Raman spectroscopy: A tool to investigate 
alpha decay damage in a $\mathrm{PuO}_{2}$ crystal lattice and determining sample age since calcination. J. Raman Spectrosc. 50, 899-901 (2019).

34. Guimbretière, G. et al. In situ Raman monitoring of $\mathrm{He}^{2+}$ irradiation induced damage in a $\mathrm{UO}_{2}$ ceramic. Appl. Phys. Lett. 103, (2013).

35. Desgranges, L., Simon, P., Martin, P., Guimbretiere, G. \& Baldinozzi, G. What Can We Learn From Raman Spectroscopy on Irradiation-Induced Defects in $\mathrm{UO}_{2}$ ? JOM 66, 2546-2552 (2014).

36. Sabathier, C. et al. In situ TEM study of temperature-induced fission product precipitation in $\mathrm{UO}_{2}$. Nucl. Instruments Methods Phys. Res. Sect. B Beam Interact. with Mater. Atoms 266, 3027-3032 (2008).

37. Barnes, R. S. \& Mazey, D. J. The nature of radiation-induced point defect clusters. Philos. Mag. 5, 1247-1253 (1960).

38. Enrique, R. A. \& Bellon, P. Compositional Patterning in Systems Driven by Competing Dynamics Of Different Length Scale. Phys. Rev. Lett. 84, 2885-2888 (2000). 